\title{
X-ray Emission as a Diagnostic for from Pseudospark-Sourced Electron Beams
}

\author{
D. Bowes ${ }^{1, a)}$, H. Yin ${ }^{1}$, W. He ${ }^{1}$, L. Zhang ${ }^{1}$, A.W. Cross ${ }^{1}$, K. Ronald ${ }^{1}$, A.D.R. Phelps ${ }^{1}$, \\ D. Chen ${ }^{2}$, P. Zhang ${ }^{2}$, X. Chen ${ }^{3}$ and D. $\mathrm{Li}^{3}$ \\ ${ }^{1}$ Department of Physics, SUPA, University of Strathclyde, Glasgow, G4 0NG, UK \\ ${ }^{2}$ Computed Tomography Lab, School of Mathematical Sciences, Capital Normal University, \\ Beijing, 100048, China \\ ${ }^{3}$ Department of Electronic Engineering, Queen Mary University of London, London, E1 4NS, \\ UK
}

X-ray emission has been achieved using an electron beam generated by a pseudospark low-pressure discharge and utilised as a diagnostic for beam detection. A $300 \mathrm{~A}, 34 \mathrm{kV}$ PS-sourced electron beam pulse of $3 \mathrm{~mm}$ diameter impacting on a $0.1 \mathrm{~mm}$-thick molybdenum target generated X-rays which were detected via the use of a small, portable X-ray detector. Clear X-ray images of a micro-sized object were captured using an X-ray photodetector. This demonstrates the inducement of proton induced X-ray emission (PIXE) not only as an indicator of beam presence but also as a future X-ray source for smallspot X-ray imaging of materials.

\section{Introduction}

Since its conception as an electron beam (e-beam) source at the university of Erlangen in 1978 [1], the pseudospark discharge has been proven to be of note both as an e-beam source and for its use in high-power switching applications [2,3]. The discharge operates within a hollow cathode / planar anode configuration on the left-hand side of the hollow cathode analog to the Paschen minimum, typically at pressures of $6.5-65 \mathrm{~Pa}$. It is notable for its fast rise time and high discharge current, as well as for the generation of a high quality electron beam in the conductive phase of the discharge. Such beams possess high current density of up to $10^{4} \mathrm{Acm}^{-2}$, high brightness up to $10^{12} \mathrm{Am}^{-2} \mathrm{rad}^{-2}$ [4] and require no guiding magnetic field. This is due to the presence of an ion channel which is formed with the generation of the electron beam $[5,6,7,8$, 9].

Because of its special discharge characteristics, the pseudospark discharge has gained considerable attention during the last 30 years, particularly with regard to its breakdown characteristics and the underlying plasma physics responsible for high current emission, and for its potential application in various fields $[10,11]$. For example, the pseudospark has been shown 
to act as a source of soft X-rays by means of X-ray fluorescence $[12,13,14]$ and X-ray bremsstrahlung [15]. In this paper, measurements of electron beams of $3 \mathrm{~mm}$ diameter from a four-gap pseudospark, impacting on a molybdenum plate (which also served as a witness plate of the electron beam) for the generation of X-rays and X-ray imaging of a micro-sized object are presented. In addition to their role in imaging, it has been found that these X-rays may function as a diagnostic tool for indicating the presence of the generated electron beam.

\section{Materials and Methods}

The X-ray experimental setup with the PS discharge is shown in figure 1. The discharge chamber itself is a four-gap PS discharge structure consisting of a planar anode, a planar cathode with a cylindrical hollow cavity, three stainless steel inter-electrodes of $3 \mathrm{~mm}$ thickness and four Perspex insulation discs of $4 \mathrm{~mm}$ thickness. Both the anode and cathode have an on-axis hole of $3 \mathrm{~mm}$ diameter. The hollow cathode cavity was made of stainless steel, having a length of $50 \mathrm{~mm}$ and outer and inner diameters of 63 and $50 \mathrm{~mm}$ respectively. These dimensions were based on the requirements of the hollow cathode effect [4]. To reduce the inductance in the discharging circuit and to act as an energy source to sustain the discharge, an external energy storage capacitor of $428 \mathrm{pF}$ was placed across the cathode and anode, consisting of three chains of capacitors in parallel. Each chain consisted of seven $15 \mathrm{kV}, 1000 \mathrm{pF}$ resin dipped ceramic capacitors connected in series. A mechanical rotary pump evacuated the experimental system from the anode end through a vacuum valve. The working gas was air and entered the chamber through a very fine controlled needle valve at the anode side and its pressure was measured by a Baratron-type vacuum gauge located at the anode together with a display meter. The hollow cathode was connected through a $30 \mathrm{M} \Omega$ charging resistor to a negative voltage source $(-100 \mathrm{kV}$, $40 \mathrm{~mA}$ dc power supply). The anode was grounded. A capacitive voltage probe of sub-ns risetime was connected to the cathode to measure the applied voltage as well as the discharge voltage while a Rogowski coil was connected next to the external storage capacitor in the discharge circuit to measure the discharge current. The measurement of the beam current was achieved using another Rogowski belt between the anode and the drift tube, as shown in figure 1.

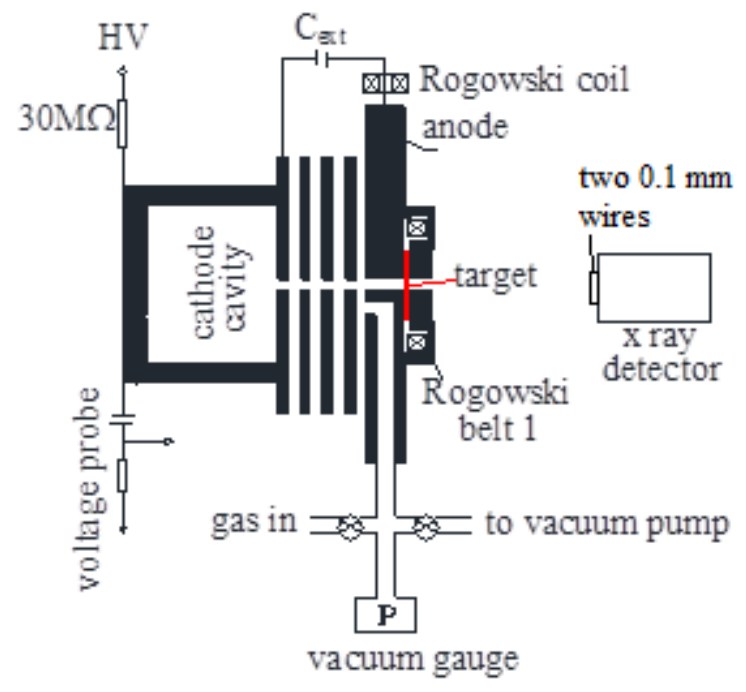

FIG. 1. Schematic experimental setup. 
The capacitive voltage probe with fast response and high load impedance was used to measure the fast risetime ( $20 \mathrm{~ns}$ ), short duration ( $20 \mathrm{~ns}$ to $50 \mathrm{~ns}$ ) discharge voltage of magnitude up to $46 \mathrm{kV}$. It is based on the derivation and then integration of the signal to be measured, which in this case was the applied as well as the discharge voltage. A pure resistive or capacitive voltage divider [16] was not suitable as it would have affected the charging circuit. In the pseudospark experiments, the voltage was measured using a $100 \mathrm{pF}$ capacitive probe with a resistance of $50 \Omega$ and a corresponding sensitivity and risetime of $1.38 \mathrm{kV} / \mathrm{V}$ and $15 \mathrm{~ns}$, respectively.

Both the discharge current and the beam current were measured by Rogowski coils as shown in Fig.1. In principle a Rogowski coil is a current transformer suitable for high frequency current measurement. Its primary is the current to be measured and the secondary often consists of many turns depending on the attenuating ratio that is required. It can operate in two modes, the self-integration or differential mode of operation with the self-integration operation having the larger sensitivity. In the pseudospark experiments, the Rogowski coils were made by winding ten turns of coil around a ferrite core. The coil was made of $0.7 \mathrm{~mm}$ diameter wire and operated in a self-integration mode with a $10 \Omega$ external resistor connected in series [17]. The calibrated sensitivity of the Rogowski coils was $1.37 \mathrm{~A} / \mathrm{V}$ with a risetime of less than $10 \mathrm{~ns}$ [18]

A 0.1 mm-thick molybdenum target for effective X-ray production was placed immediately after the PS anode. Typically, target materials must be made of metals with high melting points, and possess both good thermal conductivities and low vapour pressures [19]. Additionally, the higher the atomic number of the metal in the target, the higher the efficiency of $\mathrm{X}$-ray production [20]. The most commonly used target metal is tungsten, which has a high atomic number, a high melting point and the lowest vapour pressure of all metals. For applications in which the X-rays required are of much lower energy, the anode usually consists of molybdenum rather than tungsten. In this experiment, the molybdenum target also served as a witness plate for the PS electron beam. The cross-sectional shape and size of the electron beam from the PS could be recorded after a number of shots by repeating the PS discharge. A small, portal X-ray detector was situated within the experimental area to allow for instantaneous confirmation of the discharge while an X-ray photodetector was located opposite the anode with a micro-sized object made of two crossed metal wires of $100 \mu \mathrm{m}$ in diameter attached for X-ray photography.

The X-ray detector used was made by Photonic Science Ltd, model CoolView FDI 1:1. The available input area of the detector was $9.0 \times 6.7 \mathrm{~mm}$ with $1392 \times 1040$ pixels and a spatial resolution of $6.45 \mu \mathrm{m}$. The detector achieves digital X-ray imaging in an indirect way. An X-ray source sends a beam of X-ray photons through an object and any X-ray photons not absorbed by the object strike a layer of scintillating material that converts them into visible light photons. These visible light photons then form an image on a charge-coupled device (CCD) camera through a fibre-optic coupler with unit magnification. The scintillator of the detector is a polycrystalline layer of Gadolinium Oxysulphide with the density of $10 \mathrm{mg} / \mathrm{cm}^{2}$ and $30 \mu \mathrm{m}$ thickness optimized for resolution with X-ray energies of 5-60 keV. The integral electronic shutter can be selected from 1 millisecond to 10 minutes with automatic dark subtraction. The analog-to-digital signal converter has 12 bits digitization. An aluminium foil light exclusion membrane if required could be placed in front of the scintillator layer. 
The pseudospark was configured for operation in a free-running, as opposed to triggered, mode. At the beginning of a single shot experiment, the pseudospark chamber was evacuated through a vacuum port at the anode by a mechanical pump to $\sim 0.4 \mathrm{~Pa}$. After closing the vacuum pump by an electromagnetic valve, the cathode was charged to a given voltage and gas slowly filled the chamber by adjustment of the fine control needle valve (at a very slow rate of $d p / d t \sim$ $0.65 \mathrm{~Pa} / \mathrm{s}$ in order to obtain a uniform gas pressure) resulting in an increase in gas pressure until breakdown occurred. Critical breakdown pressure was determined by reading the display meter of the Baratron-type pressure gauge. Applied voltage, discharge current and beam current were recorded at the breakdown with a real-time digital oscilloscope. In this way, the relationship between the applied voltage and breakdown gas pressure was experimentally obtained.

Using discharge pressures acquired from single-shot discharges, a steady-state pressure was reached through the adjustment of the needle valve while pumping. when the $v$ Voltage was subsequently applied and increased until discharge occurred. In such a way, repetitive breakdown was achieved and maintained, with a repetition rate of $1 \mathrm{~Hz}$, and the X-ray detector could continuously acquire image data with each consecutive shot. X-ray images at varying discharge voltages (and consequently varying beam energies) were achieved by varying the pressure and discharge voltage.

\section{Results}

Before the molybdenum target was placed at the anode, the beam current corresponding to the discharge current and applied voltage was recorded as shown in figure 2, where an electron beam current of up to $300 \mathrm{~A}$ at $34 \mathrm{kV}$ is shown when the applied voltage was $46 \mathrm{kV}$. The larger discharge current may also be seen, where its oscillatory nature is a product of the discharge circuit, which may be thought of an analogous to a damped LRC resonator. The applied voltage is in the range of $22 \mathrm{kV}$ to $46 \mathrm{kV}$. An X-ray beam was produced by directing the electron beam generated from the 4-gap PS discharge towards the molybdenum target, which was situated at the PS anode where it acted as both vacuum seal and as witness plate for the PS electron beam, as shown in figure 3 . It shows that the beam spot is of $3 \mathrm{~mm}$ diameter. It may be assumed that the beam spot is a product of electron impact due to their increased mobility with respect to the surrounding ions. 


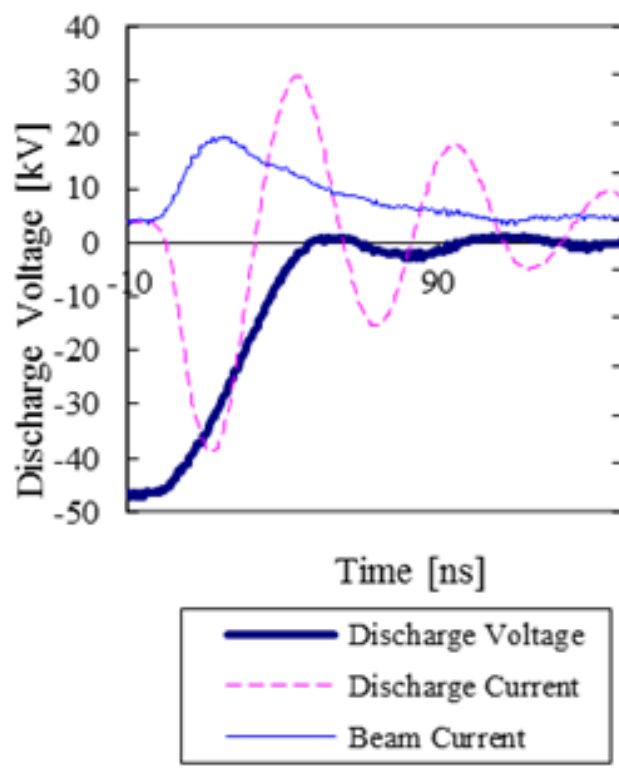

FIG. 2. Typical discharge voltage, discharge current and beam current.

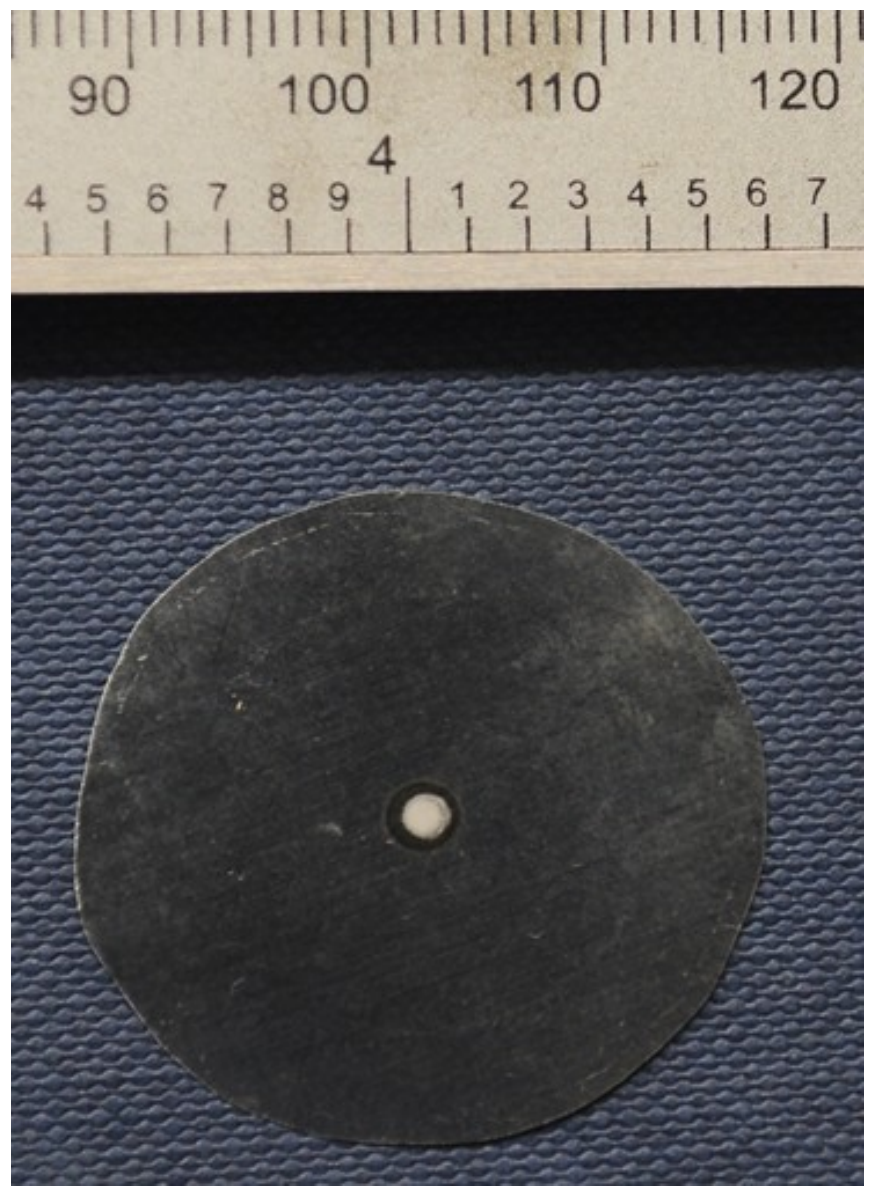

FIG. 3. Molybdenum target for X-ray generation showing the beam spot.

a) Author to whom correspondence should be addressed. Electronic mail: david.bowes@strath.ac.uk. 
The sweeping voltage induces a spread in the distribution of the generated X-ray spectrum. Using Siemens' simulator [21, 22, 23] the spectra of the X-ray photons generated from the PS-sourced electron beam hitting the anode/molybdenum target at beam voltages of 24-40 $\mathrm{kV}$ and air kerma $1 \mathrm{~Gy}$ were obtained, as shown in figure 4 . The average energy of the X-ray photons is $15.95 \mathrm{keV}$ at a beam voltage of $34 \mathrm{kV}$, with a variation of $\pm 1.5 \%$ per $\mathrm{kV}$. At present, we have not been able to verify this experimentally, though it shall be carried out in the near future, at which point a clearer comparison may be made. In order to detect such low energy Xray photons effectively, the aluminium membrane in front of the scintillator layer was taken off in the experiments. The integration time of the charge-coupled device (CCD) detector was selected to be $800 \mathrm{~ms}$, with obtained images being captured from a single discharge pulse. When the applied voltage was between $22 \mathrm{kV}$ to $46 \mathrm{kV}$, the X-ray images of the crossed metal wires were recorded with the clearest image obtained at an applied voltage of $46 \mathrm{kV}$, corresponding to the production of a $300 \mathrm{~A}, 34 \mathrm{kV}$ PS-beam. The reduced clarity of the obtained images at lower voltages effectively set a threshold voltage for the effective capture of X-ray images. An X-ray conversion efficiency of $0.15 \%$ was calculated based on the beam voltage and anode material, though this is yet to be experimentally verified and could be increased by means of a higher beam voltage or by using a target material with a higher atomic number [24]. The object, consisting of two crossed wires of $0.1 \mathrm{~mm}$ diameter, is shown in figure 5(a). An X-ray image of the object is shown in figure 5(b) when the detector was situated $5.2 \mathrm{~cm}$ from the PS anode/molybdenum target at the applied voltage of $46 \mathrm{kV}$.

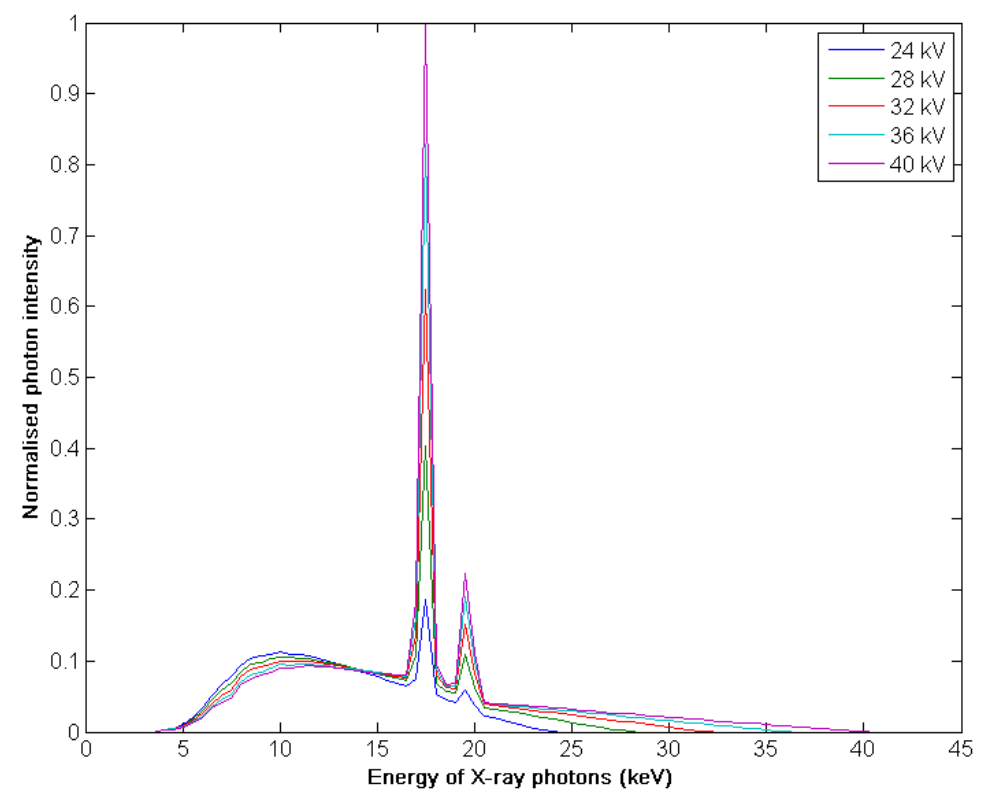

FIG. 4. Normalised Sspectrum of the X-ray photons (in arbitrary units) generated from the PSsourced electron beam at the beam voltage $34 \mathrm{kV}$ as obtained from the Siemens' simulator [18]. 


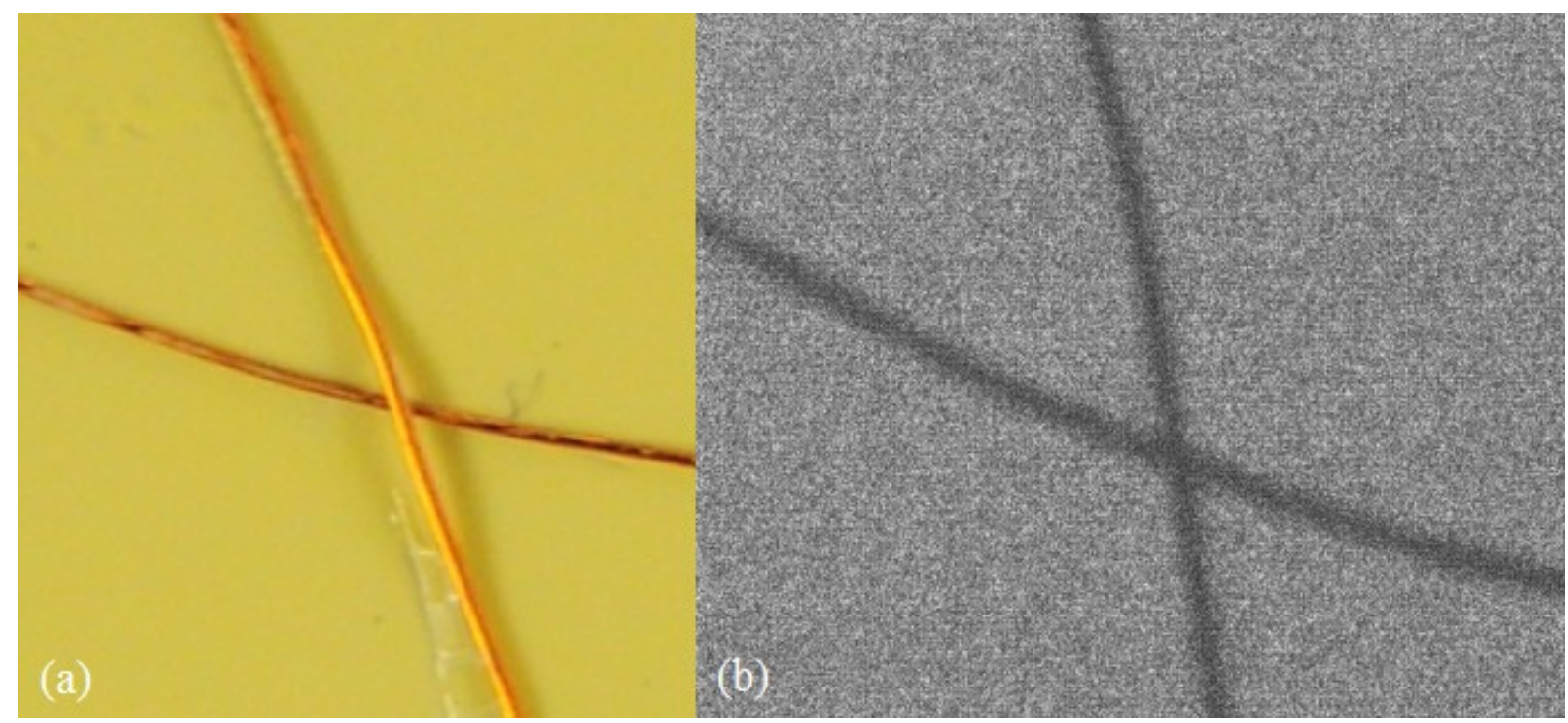

FIG. 5. (a) Object for $x$-ray image and (b) captured x-ray image of object.

The experiment has shown that a PS-sourced electron beam can be used to produce Xrays. The uses of this are two-fold. Firstly, the generation of X-rays can be used as an indicator of discharge and subsequent electron beam generation, a diagnostic method which is relatively simple, versatile and cost-effective to implement. Secondly, the great simplicity and flexibility of the PS-sourced electron beam, as well as it being a cost effective source, demonstrates its potential as a cheap, robust X-ray imaging source which may be used for sparse source computed tomography (CT) imaging such as Sparse-view CT, where a small number of PS X-ray sources and detectors simultaneously acquire data, instead of the traditional $\mathrm{CT}$ which requires the mechanical rotation of the X-ray source and detector [25]. This technology can be used in dynamic CT imaging (4D imaging) and will be the subject of future work.

\section{Conclusion}

A $3 \mathrm{~mm}$-diameter electron beam has been generated by a pseudospark discharge in a 4gap configuration. Utilising a $46 \mathrm{kV}$ charging voltage, a beam current pulse of up to $300 \mathrm{~A}$ was observed by a Rogowski coil situated adjacent to the anode. Using a molybdenum target / witness plate placed in the path of the electron beam, X-ray generation has been detected using repetitive shots at a frequency of $1 \mathrm{~Hz}$. An X-ray photodetector was located $5.2 \mathrm{~cm}$ from the target and X-ray images of a micro-sized object recorded, with the clearest images detected at an applied voltage of $46 \mathrm{kV}$, though images were also observed at lower voltages. This shows that similar electron beams generated by pseudospark discharges are good candidates for further Xray production. It is possible to scale-down a pseudospark discharge to produce micro sized electron beams to serve as a useful X-ray source for radiography of small objects for potential biological application.

\section{Acknowledgements}


The authors would like to thank the Engineering and Physical Sciences Research Council (EPSRC) for supporting this work under the grant EP/G 011087/1. P. Zhang and D. Chen were supported by the National Natural Science Foundation of China under the grant 60971131.

\section{References:}

[1] J. Christiansen and C. Schultheiss, Z. Phys. A290, 35 (1979).

[2] N. Kumar; D.K. Verma, J. Prajapati; M. Kumar; B.L. Meena; M.S. Tyagi, V. Srivastava, U. Pal, International Symposium on Vacuum Science and Technology and its application for accelerators, Journal of Physics Conference Series, 390, 012073, DOI: 10.1088/17426596/390/1/012073, (2012).

[3] N. Kumar; U.N. Pal; D.K. Verma; J. Prajapati; M. Kumar, B.L. Meena; M.S. Tyagi, V. Srivastava, Journal of Infrared and Millimeter and Terahertz Waves, 32, No 12, 1415-1423, DOI: 10.1007/s10762-011-9830-5, 2011.

[4] H. Yin, A. W. Cross, A. D. R. Phelps, D. Zhu, W. He, and K. Ronald, J. Appl. Phys. 91, 5419 (2002).

[5] M. A. Gunderson and G. Schaefer, Physics and applications of pseudosparks (NATO Advanced Study Institute, Series B: Physics vol 219) (New York: Plenum) (1990).

[6] K. Frank and J. Christiansen, IEEE Trans. Plasma Sci. 17, 748 (1989).

[7] K. K. Jain, E. Boggasch, M. Reiser and M. J. Rhee, Phys. Fluids B 2, 2487 (1990).

[8] E. Dewald, K. Frank, D. H. H. Hoffmann, R. Stark, M. Ganciu, B. N. Mandache, M. G. Nistor, A.-M. Pointu and I.-I. Popescu, IEEE Trans. Plasma Sci. 25, 272 (1997).

[9] K. Ramaswamy, W. W. Destler, and J. Rodgers, J. Appl. Phys. 83, 3514 (1998).

[10] A. W. Cross, H. Yin, W. He, K. Ronald, A. D. R. Phelps, and L. C. Pitchford, J. Phys. D: Appl. Phys. 40, 1953 (2007).

[11] H. Yin, A. W. Cross, W. He, A. D. R. Phelps, K. Ronald, D. Bowes, and C. W. Robertson, Phys. Plasmas 16, 063105 (2009).

[12] D. Bloess, I. Kamber, H. Riege, G. Bittner, V. Brückner, J. Christiansen, K. Frank, W. Hartmann, N. Lieser, C. Schultheiss, R. Seeböck, and W. Steudtner, Nucl. Instrum. Methods Phys. Res. 205, 173 (1983).

[13] C. S. Wong, H. J. Woo, and S. L. Yap, Laser Part. Beams 25, 497 (2007).

[14] M. Benk, K. Bergmann, D. Schäfer, and T. Wilhein, Opt. Lett. 33, 2359 (2008).

[15] C. Jiang, A. Kuthi, M. A. Gundersen, and W. Hartmann, Appl. Phys. Lett. 87, 131501 (2005).

[16] He W., Yin H., Phelps A.D.R., Cross A.W. and Spark S.N., 'Study of fast, high-impedance, high voltage pulse divider', Rev. Sci. Instrum., 72, pp4266-4269, 2001.

[17] R. H. Huddlestone and S. L. Leonard, "Plasma Diagnostic Techniques", Academic Press, Chapter 2, 1965

[18] H. Yin, W. He, A. W. Cross, A. D. R. Phelps, and K. Ronald, J. Appl. Phys. 90, 3212 (2001). [19] P. N. Martin; J. R. Threadgold and S. Vickers, IEEE Transactions on Plasma Science, 41, No pp2510-2515, DOI: 10.1109/TPS.2013.2275646, 2013

[20] D. C. Greene and P. C. Williams, Linear accelerators for radiation therapy (Bristol: Institute of Physics Publishing) (1997).

[21] https://w9.siemens.com/cms/oemproducts/Home/X-rayToolbox/spektrum/Pages/MamIn.aspx. [22] J. M. Boone, T. R. Fewell, and R. J. Jennings, Med. Phys. 24, 1863 (1997). 
[23] J. M. Boone, Proc. SPIE 3336, 592 (1998).

[24] P. Sprawls, Physical Principles of Medical Imaging (Medical Physics Publishing Corporation) (1995).

[25] J. Bian, J. H. Siewerdsen, X. Han, E. Y. Sidky, J. L. Prince, C. A. Pellizzari, and X. Pan, Phys. Med. Biol. 55, 6575 (2010). 